\title{
Toward improved trade-off between thermoelectric and mechanical performances in polycarbonate/single-walled carbon nanotube composite films
}

\author{
Yichuan Zhang (D) ${ }^{1}$, Liang Deng ${ }^{1}$, Haicai $\mathrm{Lv}^{1}$ and Guangming Chen (D) $^{1 凶}$
}

Polymer thermoelectric (TE) composites have witnessed explosive developments in recent years, arising from their promising prospect for lightweight flexible electronics and capability of harvesting waste-heat. In sharp contrast with intrinsically conducting polymers (CPs), the insulating thermoplastics have seldom been employed as the matrices for flexible TE composites despite their advantages of low costs, controllable melt-flowing behaviors and excellent mechanical properties. Here, we report flexible films of polycarbonate/single-walled carbon nanotube (PC/SWCNT) composites with improved trade-off between TE and mechanical performances. The SWCNTs with 1D nanostructure were dramatically aligned by PC melt-flowing under hot-pressing in the radial direction. The composite maximum power factor reaches $4.8 \pm 0.8 \mu \mathrm{W} \mathrm{m}{ }^{-1} \mathrm{~K}^{-2}$ at $10 \mathrm{wt} \%$ SWCNTs in the aligned direction, which is higher than most previously reported thermoplastics-based TE composites at the same SWCNT loading and even comparable to some intrinsically CPs and their composites. In addition, these composites display significantly higher tensile modulus and strength than CPs and their composites. This study paves an effective way to fabricate flexible films of polymer composites with simultaneously high TE and mechanical performances via judicious alignment of SWCNTs in thermoplastic polymers.

npj Flexible Electronics (2020)4:26; https://doi.org/10.1038/s41528-020-00089-2

\section{INTRODUCTION}

A recent increasing attention to flexible organic and composite thermoelectrics (TEs) mainly stems from their promising applications in complex environments to harvest low-quality heat and generate voltage upon exposure to small temperature gradients ${ }^{1-4}$. Unlike traditional inorganic TE materials (such as $\mathrm{Bi}_{2} \mathrm{Te}_{3}$ and $\mathrm{PbTe}$ ) with problems of scarcity, rigidity, high cost and element toxicity, polymers and their composites can circumvent these challenges due to their easy availability, low-cost fabrication, super flexibility, and highly-adjustable molecular structures ${ }^{4-7}$. Our group have gained some achievements in TE materials and flexible devices based on polymers and their composites ${ }^{8-10}$. To date, the polymers in flexible TEs concentrate on intrinsically conducting polymers (CPs), including poly(3,4-ethylenedioxythiophene) (PEDOT), polyaniline (PANI), poly(3-hexylthiophene) (P3HT), and polypyrrole (PPy) $)^{11-17}$. Unfortunately, compared with their boosting TE performance, little is known for the trade-off between TE and mechanical performances, although mechanical property is definitely vital for practical applications ${ }^{18}$. Moreover, unlike thermoplastics, most CPs cannot be melt-processed, and hence are limited to solution-processing method due to their strong interchain interactions. As a consequence, to solve these intractable problems are urgent for flexible TE composites.

Thermoplastic polymers are popular in daily life with distinct advantages of very low costs, cost-effective melt-processing, convenience to scale up for mass production and high mechanical properties. However, their absence of TE behavior hinders them from becoming an alternative to CPs serving as organic components for TE applications and hence the research of thermoplastics-based TE composites lags far behind the CPsbased counterparts ${ }^{19}$. Indeed, the introduction of inorganic TE constituent or carbon nanotube (CNT) can only lead to a limited increase of TE performance. For instance, the maximum power factors (defined as $P F=S^{2} \sigma, S$, and $\sigma$ are the Seebeck coefficient and the electrical conductivity, respectively) for polycarbonate (PC)/multi-walled CNT (MWCNT) and polypropylene (PP)/singlewalled CNT (SWCNT) are only $\sim 0.0008$ and $0.023 \mu \mathrm{W} \mathrm{m}{ }^{-1} \mathrm{~K}^{-220,21}$, arising from CNT physical entanglements and random distributions. Therefore, a poor trade-off between TE and mechanical properties has been obtained by simply mixing CNTs in thermoplastics. Fortunately, CNT preferred alignment is realized by flow field ${ }^{22}$, electric field ${ }^{23}$, or magnetic field ${ }^{24}$; moreover, a desired CNT distribution can be tailored by adjusting surface tension, viscosity and flowing behavior of polymer melt ${ }^{25,26}$. As a result, both electrical and mechanical properties can be improved along the aligned direction ${ }^{27}$. Therefore, an improved trade-off between TE and mechanical performances can be expected by CNT preferred alignment in thermoplastic polymers.

This work therefore utilizes the flow of molten polymer to induce SWCNT alignment in a thermoplastic (PC) matrix. Both TE performance and mechanical properties are high in the aligned SWCNT (a-SWCNT) direction of the flexible composite film. For comparison, the TE performances for the PC/a-SWCNT compsite film in the vertical direction and the flexible PC/random SWCNT ( $r$ SWCNT) composite film prepared by solvent casting are evaluated as well. The PC/a-SWCNT composite containing $10 \mathrm{wt} \%$ of SWCNTs yields a high $P F$ value of $4.8 \pm 0.8 \mu \mathrm{W} \mathrm{m}{ }^{-1} \mathrm{~K}^{-2}$ in the aligned direction, which is among the highest values for the thermoplastics-based composites at the same CNT loading and even comparable to some CPs (like PPy and P3HT) and their composites. In addition, the PC/a-SWCNT composites exhibit much higher tensile modulus and strength than CPs-based counterparts. Therefore, an improved trade-off between TE and mechanical properties has been realized for the flexible TE composites by effective SWCNT alignment.

\footnotetext{
${ }^{1}$ College of Materials Science and Engineering, Shenzhen University, 518055 Shenzhen, P. R. China. ${ }^{凶}$ email: chengm@szu.edu.cn
} 


\section{RESULTS}

Preparation and morphology observations of flexible composite films

Figure 1a presents a schematic illustration of the preparation procedures for the PC/a-SWCNT or PC/r-SWCNT composite films. The PC/a-SWCNT composites are prepared by hot-pressing the flocculation, while the PC/r-SWCNT are fabricated by simple evaporation of the suspension. Note that no distinct thermal degradation occur during the melt-preparation procedure, as ensured by thermogravimetric analysis (TGA) shown in Supplementary Fig. 1. Evidently, the composite films of both PC/a-SWCNT and PC/r-SWCNT reveal excellent flexibility, which are easily to be bent (Fig. 1b). The morphology evolution during the preparation procedure is observed by scanning electronic microscopic (SEM) images. The flocculation (Fig. 1c) displays a porous structure, and composes uniformly and randomly dispersed SWCNTs in PC matrix. During the hot-pressing procedure, SWCNTs are dramatically aligned in the radial direction, possibly due to the strong squeeze flow close to the edge of the circular sample ${ }^{28}$. Details of the SWCNT orientation are displayed in Supplementary Figs. 2 and 3. In Supplementary Fig. 2, according to the distance away from the film edge, the films are divided into 4 regions, i.e., $A-D$ regions, where the etched samples by $\mathrm{CH}_{2} \mathrm{Cl}_{2}$ show more clearly the dispersion states of SWCNTs. Evidently, the degree of the SWNCT orientation reduces in the sequence of $A>B>C>D$, which is similar to "skin-core" structure of injection-molded samples $^{29}$. Figure 1d provides a solid proof that SWCNTs adopt a high degree of alignment. In contrast, in other regions, for instance region C, both "aligned" and "randomly dispersed" SWCNTs exist simultaneously (Supplementary Fig. 3). Since the SWCNTs in region A exhibit the highest degree of orientation, it was chosen for the following TE and mechanical tests. As for the PC/a-SWCNT composites with other SWCNT contents, Supplementary Fig. 4 also confirms the significant alignment of the SWCNTs. Similar pressinginduced CNT alignment has been reported by Jiang group ${ }^{30,31}$. In sharp contrast with PC/a-SWCNT composites, a random dispersion of SWCNTs is prevalent in Fig. 1e for the PC/r-SWCNT film prepared by solvent casting. High-magnification images (Supplementary Fig. 5) distinctly display the long and curved SWCNTs are entangled. To make a more clear comparison, the two composite films are etched to remove the upper covered PC matrix. Figure $1 \mathrm{f}$ also supports the above results, i.e., the SWCNTs are significantly preferred aligned in the radical direction in PC/a-SWCNT composite films, whereas randomly dispersed in PC/r-SWCNT composite films.

\section{TE performances of flexible composite films}

The effects of SWCNT content and alignment on the composite TE performance are compared in Fig. 2a-c. Figure 2a shows that the electrical conductivities of PC/a-SWCNT (in both directions) and $\mathrm{PC} / \mathrm{r}$-SWCNT composites enhances with increasing SWCNT contents, and PC/a-SWCNT composites along the aligned direction display higher electrical conductivities than those in the vertical direction and PC/r-SWCNT composites at the same SWCNT loadings. Increased SWCNT loadings leads to higher contents of conducting constituents and more electrical conducting pathways. The reason that PC/a-SWCNT composites exhibit higher electrical conductivities along the aligned direction is explained in the following. Since SWCNTs are always dispersed in the form of SWCNT bundles in the PC matrix (see Fig. 1f), the charge transport in SWCNTs consists of intrabundle transport within each SWCNT bundle and interbundle transport among adjacent SWCNT bundles. The charge carriers transport more efficiently in intrabundles of aligned SWCNTs than interbundles ${ }^{32}$. Due to the SWCNT preferred alignment, the PC/a-SWCNT films possess more charge carriers transport via efficient intrabundle conduction along the aligned direction than the interbundle conduction in the vertical direction, leading to an electrical anisotropy for PC/aSWCNT film. Likewise, more intrabundle transports exist in PC/a-
SWCNT film in the aligned direction than those in PC/r-SWCNT film. Additionally, compared with PC/a-SWCNT film in the aligned direction, the higher contact resistances exist at interbundles for PC/a-SWCNT film in the vertical direction and PC/r-SWCNT composite film, because charge carriers have to cross more interbundle junction interfaces. Besides, in comparison with $\mathrm{PC} / \mathrm{r}$ SWCNT, PC/a-SWCNT displays a higher electrical conductivity in the vertical direction. Hot-pressing procedure may make the SWCNT contact denser compared with solvent evaporation, and results in lower contact resistances at SWCNT junctions for the PC/ a-SWCNT films along both directions, which is responsible for higher electrical conductivity of PC/a-SWCNT composite (made by hot-pressing) in the vertical direction than PC/r-SWCNT composite (obtained by vapour evaporation). Similar results were reported by Lee et al. ${ }^{33}$, where hot-pressing treatment of nanocomposite films resulted in CNT densification, and increased the electrical conductivity and the power factor. Hence, more efficient intrabundle transport and lower contact resistances at SWCNT junctions contribute to the highest electrical conductivities of PC/ a-SWCNT composite films in the aligned direction.

Figure $2 b$ shows that the Seebeck coefficients are almost independent of SWCNT content for the PC/a-SWCNT composite films at both direction and PC/r-SWCNT films. Indeed, considering experimental errors, no distinct difference exists for the Seebeck coefficients between the aligned and random samples. The independence of the Seebeck coefficients on aligned SWCNTs is consistent with previous works ${ }^{34,35}$. Consequently, the composite power factors follow the same trends of the electrical conductivity (Fig. 2c). PC/a-SWCNT composite (at $10 \mathrm{wt} \%$ of SWCNTs) shows an obviously anisotropic TE performance with $4.8 \pm 0.8$ and $3.0 \pm$ $0.8 \mu \mathrm{W} \mathrm{m} \mathrm{m}^{-1} \mathrm{~K}^{-2}$ for aligned and vertical directions, respectively. The maximum power factor $\left(4.8 \pm 0.8 \mu \mathrm{W} \mathrm{m}{ }^{-1} \mathrm{~K}^{-2}\right)$ for $\mathrm{PC} / \mathrm{a}-$ SWCNT composite in the aligned direction is higher than six times of that of the randomly dispersed (PC/r-SWCNT) sample $(0.77 \pm$ $0.02 \mu \mathrm{W} \mathrm{m}{ }^{-1} \mathrm{~K}^{-2}$ ) at the same loading. The thermal conductivities and figure of merits $(Z T)$ values for the PC/a-SWCNT composites are illustrated in Supplementary Fig. 6, also indicating a higher $Z T$ value in the aligned direction. The SWCNT alignment with 1D nanostructure greatly facilitates the transport of charge carriers, being responsible for the high TE performance in the aligned direction.

Figure $2 \mathrm{~d}$ compares the power factors of the present study with other thermoplastics-based TE composites with $10 \mathrm{wt} \%$ CNTs or CNT hybrid fillers ${ }^{21,36-42}$, the conducting polymers (PPy and P3HT) and their composites ${ }^{18,43-49}$. Thermoplastics-based composites with random CNT distributions often reveal low PF values. For example, the PC/SWCNT composites prepared by Piao et al. ${ }^{42}$ reveal similar $P F$ values with our $P C / r$-SWCNT composites at the same loadings. Obviously, the $P F$ value $\left(4.8 \pm 0.8 \mu \mathrm{W} \mathrm{m}{ }^{-1} \mathrm{~K}^{-2}\right)$ of the PC/a-SWCNT composite reported herein is higher than most previously reported thermoplastics-based TE composites at $10 \mathrm{wt}$ $\%$ CNTs. The relatively high PF values were also achieved in the PVAc or VAE latex/CNT systems ${ }^{38,39}$ with a segregated network, where CNTs are only located at the interfaces between polymer latex particles instead of being randomly distributed throughout the whole system. However, such a structure inevitably leads to CNT agglomerates at interfaces, and prevents macromolecular diffusion between the polymer domains, and hence significantly reduces the mechanical properties ${ }^{50}$. The aligned CNT structure has an obvious advantage over segregated CNT structure in terms of mechanical properties. In addition, the maximum power factor of our work is even comparable with some CPs and their composites, including nanostructured $\mathrm{PPy}^{43-45}$, PPy/CNT composites $^{18,45,46}$ and doped $\mathrm{P}_{3} \mathrm{HT}^{47-49}$. This demonstrates that superiority of utilizing 1D nanostructured SWCNTs to greatly improve the TE performance of thermoplastics-based composites. Indeed, the current $P F$ value of PC/a-SWCNT is still low for direct applications, and lower than the reported maxima for high- 
$\mathbf{a}$

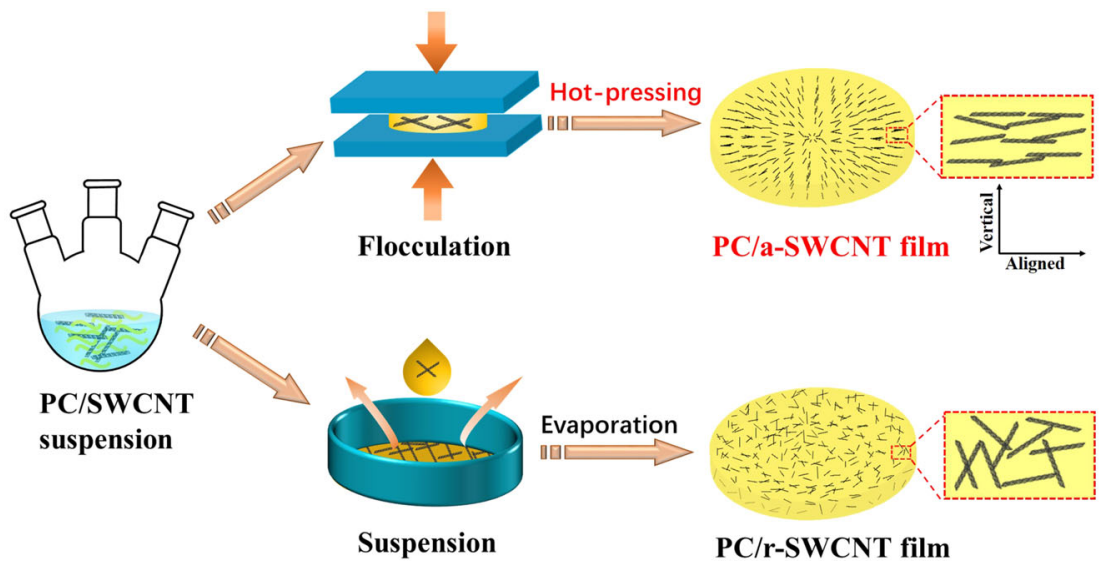

Fig. 1 The preparation and morphology of flexible TE compsoites. a Schematic illustration of the preparation procedures for PC/a-SWCNT and PC/r-SWCNT films; b Digital photographs showing excellent flexibility for PC/a-SWCNT film (a', scale bar $1 \mathrm{~cm})$ and PC/r-SWCNT film (b', scale bar $1 \mathrm{~cm}$ ); c SEM images for the flocculation (a', scale bar $5 \mu \mathrm{m}$; b', scale bar $1 \mu \mathrm{m}$ ); d SEM images for cross section of PC/a-SWCNT film (a', scale bar $5 \mu \mathrm{m}$; b', scale bar $1 \mu \mathrm{m}$ ); e SEM images for (a', scale bar $5 \mu \mathrm{m})$ cross section and (b', scale bar $5 \mu \mathrm{m})$ surface of PC/r-SWCNT film; f SEM images of etched samples by $\mathrm{CH}_{2} \mathrm{Cl}_{2}$ for (a', scale bar $1 \mu \mathrm{m}$ ) PC/a-SWCNT and (b', scale bar $\left.1 \mu \mathrm{m}\right)$ PC/r-SWCNT films. 

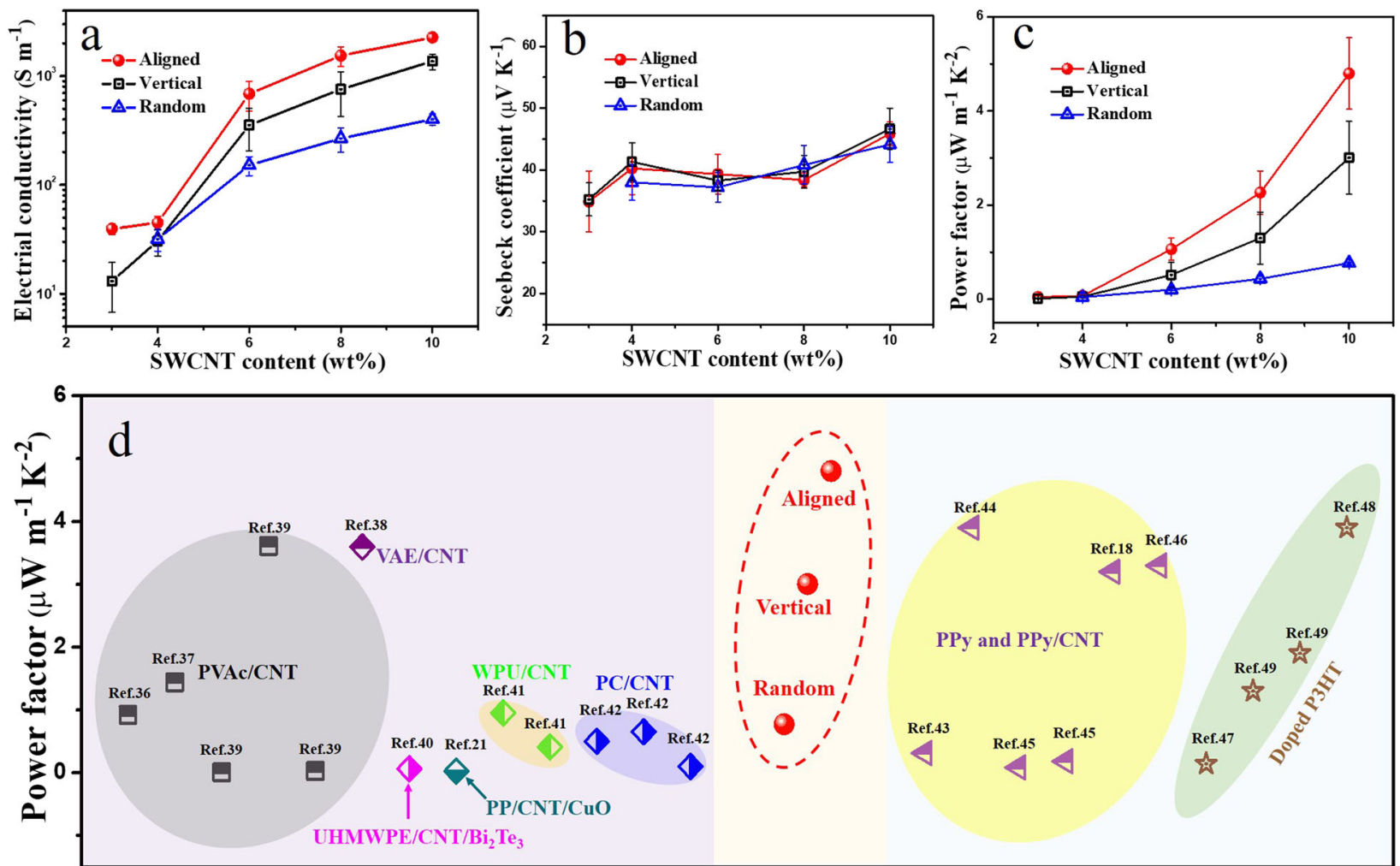

Present work

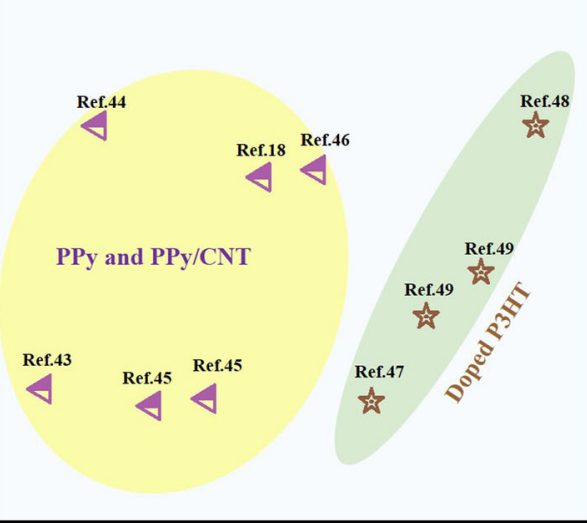

Thermoplastics-based composites

\section{Conducting polymers and their composites}

Fig. 2 The performances of TE materials. Dependencies of a electrical conductivities, b Seebeck coefficients and $\mathbf{c}$ power factors on SWCNT contents for PC/a-SWCNT (along aligned direction and vertical direction to SWCNT alignment) and PC/r-SWCNT composite films. Error bars represent standard deviation with $n \geq 5$ for each measurement. d A comparison of power factors of CNT filled thermoplastics-based composites ${ }^{21,36-42}$, conducting polymers and their CNT filled composites ${ }^{18,43-49}$. The UHMWPE/CNT/Bi ${ }_{2} \mathrm{Te}_{3}$ composite ${ }^{40}$ contains 4 wt $\%$ of CNT and $30 \mathrm{wt} \%$ of $\mathrm{Bi}_{2} \mathrm{Te}_{3}$ and PP/CNT/CuO composite ${ }^{21}$ contains $2 \mathrm{wt} \%$ of CNT and $5 \mathrm{wt} \%$ of CuO. The CNT content of other composites is fixed at $10 \mathrm{wt} \%$. Abbreviations for the mentioned polymers: PVAc is poly(vinyl acetate) latex, VAE is vinyl acetate and ethylene emulsion, UHMWPE is ultrahigh molecular weight polyethylene and WPU is waterborne polyurethane.

performance PEDOT and doped poly(2,5-bis(3-tetradecylthiophen$2-\mathrm{yl})$ thieno[3,2-b]thiophene) (PBTT $)^{51-53}$. Hence, to gain further improved TE performance for thermoplastics-based composites is still a primary focus for TE application ${ }^{19}$.

\section{Mechanical properties of flexible films}

Figure 3 a shows typical stress-strain curves of the neat PC and the PC/a-SWCNT composites in the aligned direction. Apparently, the neat PC sample exhibits a typical ductile behavior with an obvious yielding point (inset of Fig. 3a), and the PC/a-SWCNT composites show brittle fracture characteristic at high SWCNT content above $6 \mathrm{wt} \%$. Similar ductile-brittle transition was also reported for PC/ MWCNT system ${ }^{54}$. The detailed information on the tensile properties is summarized in Supplementary Table 1. A substantial increase for tensile modulus and a moderate decrease for fracture strength with increasing SWCNT contents can be seen. The high tensile modulus and strength may result from the morphology of SWCNT alignments (See SEM images in Fig. 1d) and the strong interfacial interactions (confirmed by Raman spectra in Supplementary Fig. 7) ${ }^{18}$ that leads to effective stress transfers in the composite films. On the other hand, the PC/a-SWCNT composites show low elongations at break possibly due to the aggregates at high SWCNT contents. Figure 3b depicts the relationship between mechanical properties and power factor. The tensile modulus increases with the increase of power factor. Even though the fracture strength reduces with the power factor, with power factor of $4.8 \pm 0.8 \mu \mathrm{W} \mathrm{m}{ }^{-1} \mathrm{~K}^{-2}$, the minimum value of $44.1 \pm 8.9 \mathrm{MPa}$ is still high in comparison with other TE composites ${ }^{18}$.

Improved trade-off between TE and performances

Figure 4 compares the TE and the mechanical properties between PPy/SWCNT composite, doped P3HT material and our present work. Since the PC/a-SWCNT composite shows similar elongations at break (around 2\%) with PPy/SWCNT composite ${ }^{18}$ and doped $\mathrm{P} 3 \mathrm{HT}$ material ${ }^{55}$, this mechanical parameter is not included in Fig. 4. Figure 4 clearly shows that, in addition to high TE performance, the PC/a-SWCNT composite presents superior mechanical properties over PPy/SWCNT composite (at the same SWCNT content) and doped P3HT. The tensile modulus of the PC/a-SWCNT composite is, respectively, more than 5 times and 15 times of that of the PPy/ SWCNT composite and the doped $\mathrm{P} 3 \mathrm{HT}$, respectively. In addition, the fracture strength of the PC/a-SWCNT composite is over four times and three times of that of the PPy/SWCNT composite and the doped P3HT material. This demonstrates that the present method of controlling SWCNT alignment is effective to achieve simultaneously high TE and mechanical properties for TE composites. Accordingly, it is reasonable to expect that this method can be expanded to other polymer/CNT systems, which may significantly improve the trade-off between TE and mechanical performances for polymers-based TE composites. 

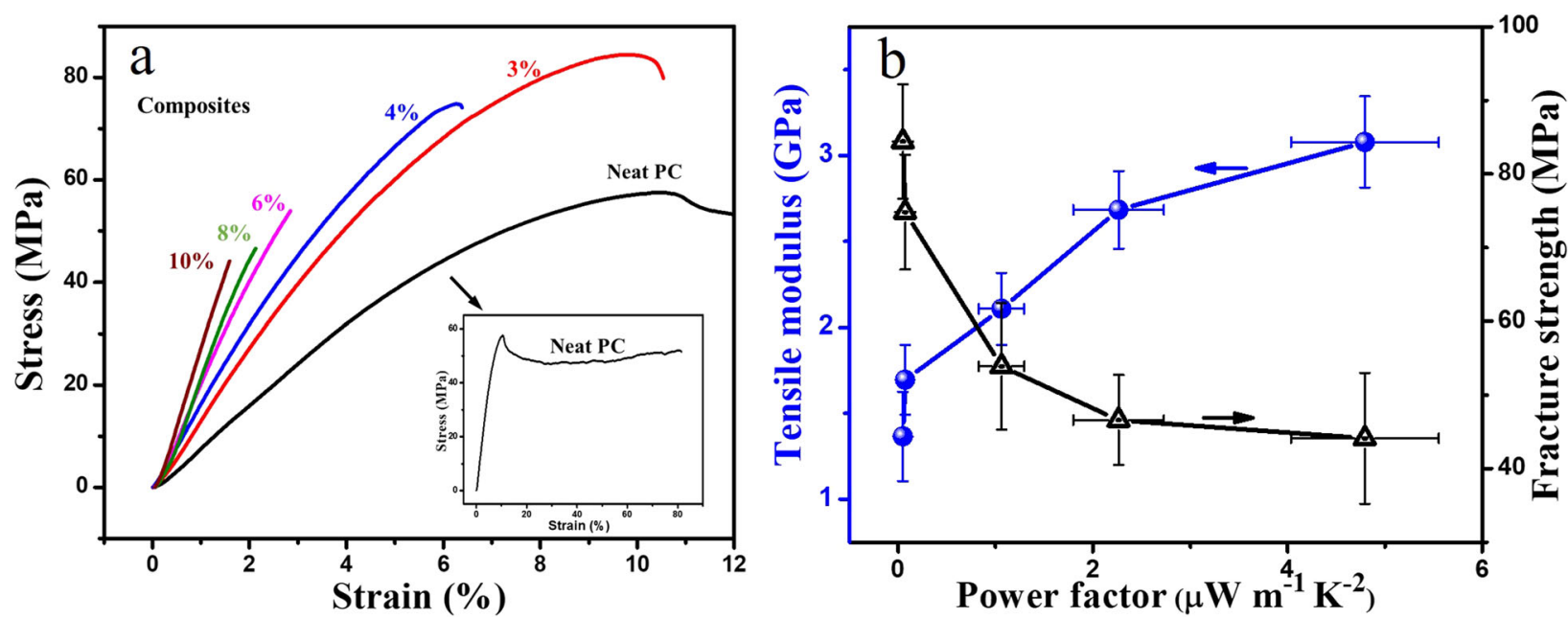

Fig. 3 The mechanical property of TE composites. a Typical stress-strain curves of PC/a-SWCNT films in the aligned direction as a function of SWCNT contents; The inset shows the whole stress-strain curve of neat PC for comparison. $\mathbf{b}$ The mechanical properties versus the power factor for PC/a-SWCNT films. Error bars represent standard deviation with $n \geq 5$ for each measurement.

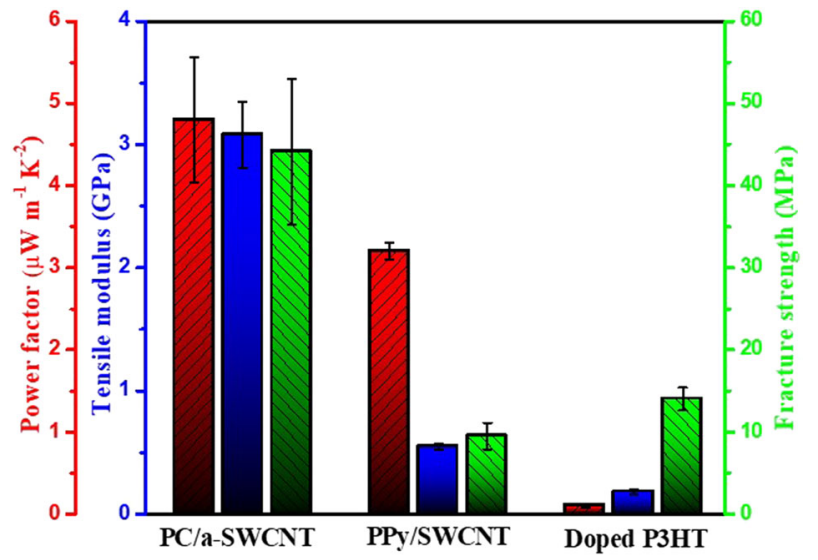

Fig. 4 The comparison of TE and mechanical performances. A comparison of power factors, tensile modulus, fracture strength of PC/a-SWCNT composite in the aligned direction (SWCNT: $10 \mathrm{wt} \%$ ), PPy/SWCNT composite (SWCNT: $10 \mathrm{wt} \%)^{18}$ and 2,3,5,6-tetrafluoro7,7,8,8-tetracyanoquinodimethane (F4TCNQ) doped P3HT material ${ }^{55}$. Note that the fracture strength of the doped $\mathrm{P} 3 \mathrm{HT}$ material was not given in ref. ${ }^{55}$. and its true value is even lower than the value here that is estimated by neat P3HT material. Error bars in PC/a-SWCNT composite represent standard deviation with $n \geq 5$ for each measurement, and error bars in PPy/SWCNT and P3HT materials are from the values in refs. ${ }^{18,55}$.

\section{DISCUSSION}

In summary, we report the fabrication of flexible films of PC/aSWCNT composites with SWCNT preferred alignment in the radial direction induced by polymer melt flow during hot-pressing. PC/aSWCNT composite films display an abrupt increase of the electrical conductivity and an almost independency of the Seebeck coefficient with increasing SWCNT content. As a result, the maximum power factor reaches $4.8 \pm 0.8 \mu \mathrm{W} \mathrm{m}{ }^{-1} \mathrm{~K}^{-2}$ along the aligned direction at $10 \mathrm{wt} \%$ of SWCNT, much higher than that in vertical direction and the PC/r-SWCNT film at the same SWCNT content. This value is higher than that of most thermoplasticsbased TE composites at $10 \mathrm{wt} \%$ of SWCNTs, and even comparable to conducting polymers and their composites. More importantly, the mechanical test confirms that the PC/a-SWCNT composite show higher tensile modulus and strength than PPy/SWCNT and doped P3HT, indicating an improved trade-off between TE and mechanical performances. Although the current TE performance of PC/SWCNT compsite is still lower than the reported maxima for some CPs and their composites, this study paves an effective way to achieve an improved trade-off between TE and mechanical performances by effectively inducing CNT preferred alignment and rational utilization of the 1D nanostructure of CNTs during melt-processing. The strategy may help overcome the intractable trade-off issue between TE and mechanical performances for CPsbased composites, and thus benefits the development of flexible polymer TE composites and widens versatile applications of flexible waste-heat harvesting electronics, etc.

\section{METHODS}

Chemicals and materials

PC (model PC2407) was provided by Covestro Polymers (China) Co. Ltd., and SWCNTs (diameter $<2 \mathrm{~nm}$, length $>5 \mathrm{um}$, purity $>90.0 \%$ ) with a commercial trademark of NTP SWNT-2 were purchased by Shenzhen Nanotech Port Co. Ltd., China. Both the PC and SWCNTs were dried $80^{\circ} \mathrm{C}$ for $24 \mathrm{~h}$ before use. All other reagents, including dichloromethane $\left(\mathrm{CH}_{2} \mathrm{Cl}_{2}\right.$, A.R.), anhydrous ethanol (A.R.) and deionized water, were used as received during the preparation procedure without any further purification.

\section{Preparation of PC/SWCNT composite films}

The preparation procedure of PC/a-SWCNT film was schematically illustrated in Fig. 1a. A solution method was utilized to prepare PC/SWCNT flocculation. Here, the PC/SWCNT (90/10 wt/wt) composite fabrication is taken as an example. First, SWCNT powder $(0.5 \mathrm{~g})$ was dispersed to $\mathrm{CH}_{2} \mathrm{Cl}_{2}$ $(200 \mathrm{~mL}$ ) with ultrasonic oscillation (Ultrasonic Homogenizer SCIENTZ-JY92HDN) at room temperature for $40 \mathrm{~min}$. Simultaneously, $4.5 \mathrm{~g}$ of PC was completely dissolved in $\mathrm{CH}_{2} \mathrm{Cl}_{2}(100 \mathrm{~mL})$ at $60^{\circ} \mathrm{C}$ in a three-necked bottle. The SWCNT/ $\mathrm{CH}_{2} \mathrm{Cl}_{2}$ suspension was then drop-wisely loaded into the three-necked bottle containing $\mathrm{PC} / \mathrm{CH}_{2} \mathrm{Cl}_{2}$ solution with a condenser and continuous stirring in an ultrasound bath at $50{ }^{\circ} \mathrm{C}$, and the mixture was ultrasonicated for another $20 \mathrm{~min}$. The $\mathrm{CH}_{2} \mathrm{Cl}_{2}$ here was selected as solvent for SWCNT in present work based on the facts that it has better ability to debundle and disperse the SWCNTs than water, ethanol and toluene ${ }^{56}$, and moreover the $\mathrm{CH}_{2} \mathrm{Cl}_{2}$ solubility to $\mathrm{PC}$ can almost remain constant with addition of SWCNT/ $\mathrm{CH}_{2} \mathrm{Cl}_{2}$ suspension, which guarantees PC does not precipitate out in this mixing procedure. The resultant mixture was flocculated in ethanol and dried in vacuum oven at $80^{\circ} \mathrm{C}$ for $24 \mathrm{~h}$. Finally, the dried flocculation was molten at $280^{\circ} \mathrm{C}$ for $3 \mathrm{~min}$, then hot-pressed quickly for $5 \mathrm{~min}$ and a nearly circular sample was obtained with the subsequent cold-pressing. It should be noted that the hot-pressing mold was not adopt to enable the molten polymer to flow as possible as it can, 
which was significantly different from the common compression with mold that restricts the flow and produces several mm-thick isotropic sheets ${ }^{57}$. Therefore, a thickness around $80 \mu \mathrm{m}$ and a radius around $120 \mathrm{~mm}$ was achieved for the nearly circular sample. The SWCNTs were expected to align with the flow of PC melt under hot-pressing in the radial direction. The PC/a-SWCNT film samples of around $15 \times 5 \mathrm{~mm}^{2}$ for TE measurements or around $30 \times 10 \mathrm{~mm}^{2}$ for mechanical measurements were obtained by cutting the edge of the nearly circular sample along the radial direction. The other PC/a-SWCNT samples with different SWCNT contents were prepared in the same manner as described above.

For comparison, the PC/r-SWCNT films were also prepared by solvent casting to highlight the role of SWCNT alignment in the enhancement of TE performance. The preparation procedure of PC/r-SWCNT film was also illustrated in Fig. 1a. The obtained PC/SWCNT suspension was directly poured onto a glass petri dish, which was cleaned with detergent, deionized water and isopropyl alcohol, and then dried by a flux of nitrogen before use. The PC/r-SWCNT composite film was obtained after evaporation of $\mathrm{CH}_{2} \mathrm{Cl}_{2}$ at ambient conditions and subsequent dry at $80^{\circ} \mathrm{C}$ in vacuum oven for $24 \mathrm{~h}$. This procedure was similar to that of Piao et al. ${ }^{42}$ to produce PC/r-SWCNT composites.

\section{Morphological and structural characterization}

In order to observe the distribution of SWCNTs more clearly, the PC matrix of was etched by $\mathrm{CH}_{2} \mathrm{Cl}_{2}$. The morphological observations of PC/SWCNT flocculation, PC/a-SWCNT, PC/r-SWCNT and etched samples were observed with a high resolution scanning electronic microscope (SEM, FEI APREO S). Prior to SEM observation, the sample surfaces were sputtered with platinum gold. TGA studies were performed on TA TGA-Q50 thermal analysis system in nitrogen from room temperature to $780^{\circ} \mathrm{C}$ at a heating rate of $10^{\circ} \mathrm{C} \mathrm{min}-1$. The Raman spectra were recorded on a Raman spectrometer (InVia) with a regular mode at a wavelength of $532 \mathrm{~nm}$.

\section{TE performance measurements}

The Seebeck coefficients and electrical conductivities were measured at room temperature by a commercially available Thin-Film Thermoelectric Parameter Test System (MRS-3RT, Wuhan Joule Yacht Science \& Technology Co., Ltd.) with a quasi-steady-state mode. The rectangle sample of around $15 \times 5 \mathrm{~mm}^{2}$ was used for the measurements of electrical conductivities and the Seebeck coefficients and the thickness of the sample is around $80 \mu \mathrm{m}$. The parallel thermal conductance method was performed to measure the thermal conductivities of PC/a-SWCNT films by a home-made apparatus and its schematic diagram was described in previous work ${ }^{58}$. For the PC/a-SWCNT samples, the TE parameters were tested along the aligned SWCNT direction and the direction vertical to SWCNT alignment (the testing directions were illustrated in the Fig. 1a). At least five samples were measured and an average value was used.

\section{Mechanical property tests}

Mechanical tensile tests of PC/a-SWCNT films were conducted along SWCNT alignment direction using the CMT4204 testing instrument with speed of $3 \mathrm{~mm} \mathrm{mim}^{-1}$ at $25^{\circ} \mathrm{C}$. The rectangle sample of $10 \times 30 \mathrm{~mm}^{2}$ was used for the mechanical tensile tests and the thickness of the sample is around $80 \mu \mathrm{m}$. The tensile properties of a neat PC film were also measured for comparison.

\section{DATA AVAILABILITY}

The authors declare that the data supporting the findings of this study are available within the paper (and its supplementary information files). Data are available upon request.

Received: 4 May 2020; Accepted: 11 September 2020; Published online: 02 October 2020

\section{REFERENCES}

1. Chen, G., Li, Y., Bick, M. \& Chen, J. Smart textiles for electricity generation. Chem. Rev. 120, 3668-3720 (2020).

2. Han, S. et al. Thermoelectric polymer aerogels for pressure-temperature sensing applications. Adv. Funct. Mater. 27, 1703549 (2017).
3. Qu, D., Li, X., Wang, H. \& Chen, G. Assembly strategy and performance evaluation of flexible thermoelectric devices. Adv. Sci. 6, 1900584 (2019).

4. Zhao, W. et al. Flexible carbon nanotube papers with improved thermoelectric properties. Energy Environ. Sci. 5, 5364-5369 (2012).

5. Wang, Q. et al. Hexagonal boron nitride nanosheets doped pyroelectric ceramic composite for high-performance thermal energy harvesting. Nano Energy 60, 144-152 (2019).

6. $\mathrm{Pu}, \mathrm{S}$. et al. Promoting energy efficiency via a self-adaptive evaporative cooling hydrogel. Adv. Mater. 32, 1907307 (2020).

7. Chen, G., Xu, W. \& Zhu, D. Recent advances in organic polymer thermoelectric composites. J. Mater. Chem. C. 5, 4350-4360 (2017).

8. Wu, G. et al. Exploring high-performance $\mathrm{n}$-type thermoelectric composites using amino-substituted rylene dimides and carbon nanotubes. ACS Nano 11, 5746-5752 (2017).

9. Fan, W., Liang, L., Zhang, B., Guo, C. \& Chen, G. PEDOT thermoelectric composites with excellent power factors prepared by 3-phase interfacial electropolymerization and carbon nanotube chemical doping. J. Mater. Chem. A 7, 13687-13694 (2019).

10. Qu, D., Huang, X., Li, X., Wang, H. \& Chen, G. Annular flexible thermoelectric devices with integrated-module architecture. npj Flex. Electron. 4, 1 (2020).

11. Russ, B., Glaudell, A., Urban, J. J., Chabinyc, M. L. \& Segalman, R. A. Organic thermoelectric materials for energy harvesting and temperature control. Nat. Rev. Mater. 1, 16050 (2016).

12. Fan, W., Zhang, Y., Guo, C.-Y. \& Chen, G. Toward high thermoelectric performance for polypyrrole composites by dynamic 3-phase interfacial electropolymerization and chemical doping of carbon nanotubes. Compos. Sci. Technol. 183, 107794 (2019).

13. Yao, $\mathrm{H}$. et al. Recent development of thermoelectric polymers and composites. Macromol. Rapid Commun. 39, 1700727 (2018).

14. Yao, C.-J., Zhang, H.-L. \& Zhang, Q. Recent progress in thermoelectric materials based on conjugated polymers. Polymers 11, 107 (2019).

15. Xie, J., Zhao, C. E., Lin, Z. Q., Gu, P. Y. \& Zhang, Q. Nanostructured conjugated polymers for energy-related applications beyond solar cells. Chem. Asian J. 11, 1489-1511 (2016).

16. $\mathrm{Pu}, \mathrm{S}$. et al. Thermogalvanic hydrogel for synchronous evaporative cooling and low-grade heat energy harvesting. Nano Lett. 20, 3791-3797 (2020).

17. Wang, X., Suwardi, A., Lim, S. L., Wei, F. \& Xu, J. Transparent flexible thin-film p-n junction thermoelectric module. npj Flex. Electron. 4, 19 (2020).

18. Liang, L., Gao, C., Chen, G. \& Guo, C.-Y. Large-area, stretchable, super flexible and mechanically stable thermoelectric films of polymer/carbon nanotube composites. J. Mater. Chem. C. 4, 526-532 (2016).

19. Zhang, Y., Zhang, Q. \& Chen, G. Carbon and carbon composites for thermoelectric applications. Carbon Energy 2, 408-436 (2020).

20. Liebscher, M. et al. Influence of the MWCNT surface functionalization on the thermoelectric properties of melt-mixed polycarbonate composites. Compos. Sci. Technol. 101, 133-138 (2014).

21. Luo, J. et al. Polypropylene-based melt mixed composites with singlewalled carbon nanotubes for thermoelectric applications: Switching from p-type to ntype by the addition of polyethylene glycol. Polymer 108, 513-520 (2017).

22. Zhang, Q., Vichchulada, P., Cauble, M. A. \& Lay, M. D. Percolation in networks of aligned SWNTs formed with laminar flow deposition. J. Mater. Sci. 44, 1206-1211 (2009).

23. Zhang, Y., Zheng, D., Pang, H., Tang, J.-H. \& Li, Z.-M. The effect of molecular chain polarity on electric field-induced aligned conductive carbon nanotube network formation in polymer melt. Compos. Sci. Technol. 72, 1875-1881 (2012).

24. Steinert, B. W. \& Dean, D. R. Magnetic field alignment and electrical properties of solution cast PET-carbon nanotube composite films. Polymer 50, 898-904 (2009).

25. Zhang, Y. et al. Spreading dynamics of molten polymer drops on glass substrates. Langmuir 33, 8447-8454 (2017).

26. Göldel, A., Kasaliwal, G. \& Pötschke, P. Selective localization and migration of multiwalled carbon nanotubes in blends of polycarbonate and poly (styreneacrylonitrile). Macromol. Rapid Commun. 30, 423-429 (2009).

27. Wang, X. et al. Mechanical and electrical property improvement in CNT/Nylon composites through drawing and stretching. Compos. Sci. Technol. 71, 1677-1683 (2011).

28. Dweib, M. A. \& Brádaigh, C. M. Ó. Compression molding of glass reinforced thermoplastics: Modeling and experiments. Polym. Compos. 21, 832-845 (2000).

29. Tiusanen, J., Vlasveld, D. \& Vuorinen, J. Review on the effects of injection moulding parameters on the electrical resistivity of carbon nanotube filled polymer parts. Compos. Sci. Technol. 72, 1741-1752 (2012).

30. Chen, J. et al. Strong anisotropy in thermoelectric properties of CNT/PANI composites. Carbon 114, 1-7 (2017).

31. Chen, J. et al. Revealing the anisotropy in thermoelectric transport performances in CNT/PANI composites. Synth. Met. 239, 13-21 (2018). 
32. Jung, J. et al. Efficient debundling of few-walled carbon nanotubes by wrapping with donor-acceptor polymers for improving thermoelectric properties. ACS Appl. Mater. Interfaces 11, 47330-47339 (2019).

33. Lee, W., Kang, Y. H., Lee, J. Y., Jang, K.-S. \& Cho, S. Y. Hot-pressing for improving performance of CNT/conjugated polymer thermoelectric films and power generators. Mater. Today Commun. 10, 41-45 (2017).

34. Fukuhara, K. et al. Isotropic Seebeck coefficient of aligned single-wall carbon nanotube films. Appl. Phys. Lett. 113, 243105 (2018).

35. Hone, J. et al. Electrical and thermal transport properties of magnetically aligned single wall carbon nanotube films. Appl. Phys. Lett. 77, 666-668 (2000).

36. Yu, C., Kim, Y. S., Kim, D. \& Grunlan, J. C. Thermoelectric behavior of segregatednetwork polymer nanocomposites. Nano Lett. 8, 4428-4432 (2008).

37. Kim, Y. S., Kim, D., Martin, K. J., Yu, C. \& Grunlan, J. C. Influence of stabilizer concentration on transport behavior and thermopower of CNT-filled latex-based composites. Macromol. Mater. Eng. 295, 431-436 (2010).

38. Kim, D., Kim, Y., Choi, K., Grunlan, J. C. \& Yu, C. Improved thermoelectric behavior of nanotube-filled polymer composites with poly (3, 4-ethylenedioxythiophene) poly (styrenesulfonate). ACS Nano 4, 513-523 (2010).

39. Moriarty, G. P., Wheeler, J. N., Yu, C. \& Grunlan, J. C. Increasing the thermoelectric power factor of polymer composites using a semiconducting stabilizer for carbon nanotubes. Carbon 50, 885-895 (2012).

40. Pang, $\mathrm{H}$. et al. Thermoelectric behaviour of segregated conductive polymer composites with hybrid fillers of carbon nanotube and bismuth telluride. Mater Lett. 107, 150-153 (2013).

41. Wu, Q. \& Hu, J. Waterborne polyurethane based thermoelectric composites and their application potential in wearable thermoelectric textiles. Compos. Part B Eng. 107, 59-66 (2016).

42. Piao, M., Kim, G., Kennedy, G. P., Roth, S. \& Dettlaff-Weglikowska, U. Thermoelectric properties of single walled carbon nanotube networks in polycarbonate matrix. Phys. Status Solidi B 250, 1468-1473 (2013).

43. $\mathrm{Wu}, \mathrm{J}$. et al. Polypyrrole nanotube film for flexible thermoelectric application. Synth. Met. 196, 173-177 (2014).

44. Culebras, M., Uriol, B., Gómez, C. M. \& Cantarero, A. Controlling the thermoelectric properties of polymers: application to PEDOT and polypyrrole. Phys. Chem. Chem. Phys. 17, 15140-15145 (2015)

45. Wang, J., Cai, K., Shen, S. \& Yin, J. Preparation and thermoelectric properties of multi-walled carbon nanotubes/polypyrrole composites. Synth. Met. 195, 132-136 (2014).

46. Liang, L., Chen, G. \& Guo, C.-Y. Enhanced thermoelectric performance by selfassembled layered morphology of polypyrrole nanowire/single-walled carbon nanotube composites. Compos. Sci. Technol. 129, 130-136 (2016).

47. Xuan, Y. et al. Thermoelectric properties of conducting polymers: the case of poly (3-hexylthiophene). Phys. Rev. B 82, 115454 (2010).

48. $\mathrm{He}, \mathrm{M}$. et al. Thermopower enhancement in conducting polymer nanocomposites via carrier energy scattering at the organic-inorganic semiconductor interface. Energy Environ. Sci. 5, 8351 (2012).

49. Qu, S. et al. Highly anisotropic P3HT films with enhanced thermoelectric performance via organic small molecule epitaxy. NPG Asia Mater. 8, e292 (2016).

50. Pang, H., Xu, L., Yan, D.-X. \& Li, Z.-M. Conductive polymer composites with segregated structures. Prog. Polym. Sci. 39, 1908-1933 (2014).

51. Fan, Z., Du, D., Guan, X. \& Ouyang, J. Polymer films with ultrahigh thermoelectric properties arising from significant seebeck coefficient enhancement by ion accumulation on surface. Nano Energy 51, 481-488 (2018).

52. Patel, S. N. et al. Morphology controls the thermoelectric power factor of a doped semiconducting polymer. Sci. Adv. 3, e1700434 (2017).

53. Vijayakumar, V. et al. Bringing conducting polymers to high order: toward conductivities beyond $10^{5} \mathrm{~S} \mathrm{~cm}^{-1}$ and thermoelectric power factors of $2 \mathrm{mw} \mathrm{m}^{-1}$ $\mathrm{K}^{-2}$. Adv. Energy Mater. 9, 1900266 (2019).

54. Pande, S., Chaudhary, A., Patel, D., Singh, B. P. \& Mathur, R. B. Mechanical and electrical properties of multiwall carbon nanotube/polycarbonate composites for electrostatic discharge and electromagnetic interference shielding applications. RSC Adv. 4, 13839-13849 (2014).

55. Kiefer, D. et al. A solution-doped polymer semiconductor: insulator blend for thermoelectrics. Adv. Sci. 4, 1600203 (2017).

56. Kharissova, O. V., Kharisov, B. I. \& de Casas Ortiz, E. G. Dispersion of carbon nanotubes in water and non-aqueous solvents. RSC Adv. 3, 24812-24852 (2013).

57. Gao, X. et al. Preparation of high performance conductive polymer fibres from double percolated structure. J. Mater. Chem. 21, 6401-6408 (2011).

58. Wang, H., Chu, W. \& Chen, G. A brief review on measuring methods of thermal conductivity of organic and hybrid thermoelectric materials. Adv. Electron. Mater. 5, 1900167 (2019).

\section{ACKNOWLEDGEMENTS}

This work was financially supported by Guangdong Basic and Applied Basic Research Foundation (No. 2019A1515111196) and National Natural Science Foundation of China (No. 51973122). We also acknowledge the SEM characterization provided by Instrumental Analysis Center of Shenzhen University (Lihu Campus).

\section{AUTHOR CONTRIBUTIONS}

G.C. designed and supervised the project. Y.Z. performed the material preparation characterization, and result discussion. L.D. participated in material preparation and result discussion. H.L. participated in material preparation and data analysis. Y.Z. and G.C. wrote the manuscript. All authors provided comments for the manuscript.

\section{COMPETING INTERESTS}

The authors declare no competing interests.

\section{ADDITIONAL INFORMATION}

Supplementary information is available for this paper at https://doi.org/10.1038/ s41528-020-00089-2.

Correspondence and requests for materials should be addressed to G.C.

Reprints and permission information is available at http://www.nature.com/ reprints

Publisher's note Springer Nature remains neutral with regard to jurisdictional claims in published maps and institutional affiliations.

\begin{abstract}
Open Access This article is licensed under a Creative Commons Attribution 4.0 International License, which permits use, sharing, adaptation, distribution and reproduction in any medium or format, as long as you give appropriate credit to the original author(s) and the source, provide a link to the Creative Commons license, and indicate if changes were made. The images or other third party material in this article are included in the article's Creative Commons license, unless indicated otherwise in a credit line to the material. If material is not included in the article's Creative Commons license and your intended use is not permitted by statutory regulation or exceeds the permitted use, you will need to obtain permission directly from the copyright holder. To view a copy of this license, visit http://creativecommons. org/licenses/by/4.0/.
\end{abstract}

(c) The Author(s) 2020 